\title{
Maturational effect on physical capacities and anabolic hormones in under-16 elite footballers: a cross-sectional study
}

\author{
Ebrahim Eskandarifard ${ }^{1} \cdot$ Rui Silva $^{2} \cdot$ Hadi Nobari $^{3,4}$ (C) Filipe Manuel Clemente $^{2,5}$. Jorge Pérez-Gómez ${ }^{3}$. \\ António José Figueiredo ${ }^{6}$
}

Received: 8 March 2021 / Accepted: 11 June 2021 / Published online: 20 December 2021

(c) The Author(s) 2021

\begin{abstract}
Purpose The purposes of this study were to describe the fitness and hormonal levels according to playing time (PT) (i.e., PT during season less (PT1) or more (PT2) than 50\% of the total time) and maturation level (ML) (i.e., normal (ML1) and early maturity levels (ML2)), and to analyze the differences between groups for the measures of aerobic capacity, anaerobic power, power performance, and hormonal concentrations.

Methods Twenty-four youth footballers of a U16 team participated in this study. Anthropometric measures, maturity status, growth hormone, insulin-like growth factor (IGF-1), maximal oxygen uptake, fatigue index, and countermovement jump were collected.

Results Significant differences were found between both PT and ML groups for maturational status, aerobic capacity, power performance, and IGF1 concentrations. The interaction of PT and ML revealed significant differences for maturity offset and power performance. When using the skeletal age as a covariant, the previously significant differences found were reduced only to the fatigue index measure.

Conclusions The response variables analyzed in the present study seem to be influenced by PT and ML. This must be considered when planning training, and coaches must be sensible to these effects as they may assume a preponderant role in PT.
\end{abstract}

Keywords Soccer $\cdot$ Motor development $\cdot$ Performance $\cdot$ Growth

Hadi Nobari

hadi.nobari1@gmail.com

$\triangle$ Jorge Pérez-Gómez jorgepg100@gmail.com

1 Department of Exercise Physiology, Faculty of Sport Sciences, University of Isfahan, 81746-7344 Isfahan, Iran

2 Escola Superior Desporto e Lazer, Instituto Politécnico de Viana do Castelo, Rua Escola Industrial e Comercial de Nun'Álvares, 4900-347 Viana do Castelo, Portugal

3 HEME Research Group, Faculty of Sport Sciences, University of Extremadura, 10003 Cáceres, Spain

4 Sports Scientist, Sepahan Football Club, Isfahan, Iran

5 Delegação da Covilhã, Instituto de Telecomunicações, 1049-001 Lisboa, Portugal

6 Faculty of Sport Sciences and Physical Education, University of Coimbra, Coimbra, Portugal

\section{Introduction}

Football (soccer) has evolved into a more physically, technical, and tactical demanding sport over the past few years [1]. Indeed, this fact has been corroborated by studies using data collected via modern devices, such as global positioning systems, enabling to reveal that elite football teams are dealing with greater match demands [2]. As such, this information can be used by coaches and scouts for talent identification and player selection [3]. Considering the approaches for talent identification and player selection, it has been documented that coaches tend to use unidimensional settings, where the levels of strength, speed, and morphological characteristics of young football athletes are prioritized [4].

During youth, especially from 10 to 15 years, it is expected that children present significant variations in maturation status [5, 6]. In fact, great differences in maturation status (biological age) are commonly seen within children of the same age, based on the date of birth (chronological age) [7]. Football academies usually organize youth teams 
by grouping athletes according to their chronological age, and consequently, these practices result in greater withinsubjects variation in maturity status [8]. From a practical perspective, late-maturing footballers must deal with an unfair fight, considering that early maturing players are commonly taller, heavier, and outperform their late-maturing colleagues, as well as being preferred by coaches for selection [9]. For those reasons, it is of paramount importance to estimate the maturation level (ML) of young footballers. Although this can be done through the Fels method [10], which is the most valid and reliable method to calculate the skeletal age, it requires equipment that may not be reachable for some sport contexts. However, coaches have simple and inexpensive tools to assess the maturation status of athletes at their disposal. It is possible to predict how far from the peak height velocity (PHV) children are, or if they already reached it, through anthropometric measures and a simple calculation [11].

Aerobic capacity has been considered an important performance determinant of adult elite footballers, where values of maximal oxygen consumption $\left(\mathrm{VO}_{2 \max }\right)$ can go up to $69 \mathrm{ml} \mathrm{kg}^{-1} \mathrm{~min}^{-1}[12,13]$. And it is commonly assessed via incremental tests to exhaustion, such as the yo-yo intermittent recovery (YYIR) levels 1 and 2 [14]. On the other hand, adolescent footballers have demonstrated an average level of $\mathrm{VO}_{2 \max }$ of $48 \mathrm{ml} \mathrm{kg}^{-1} \mathrm{~min}^{-1}$, which can be slightly different depending on the level of competition (elite or non-elite) and the maturity of the players [15-17]. In the same line, anaerobic power has an important role in football due to the repeated short high-intensity actions that are required during training and matches $[17,18]$. This highlights the importance of assessing the repeated sprint ability (RSA) and their related fatiguing effects (fatigue index), in adolescent footballers $[19,20]$. Lower limb explosive power is also one of the key-determinants for achieving success in elite football performance, and it is usually assessed using vertical jumps such as the countermovement jump (CMJ) [15]. Given the abovementioned physical determinants, it is fundamental to highlight the between-player variations and status of those physical aspects in critical stages of human maturation, such as puberty. In fact, it has been documented that early maturing players have greater physical characteristics and capabilities than late maturers [7]. Also, considering the typical hormonal changes during puberty, associations between the activity of some hormones, such as growth hormone (GH) and insulin-like growth factor (IGF1) with physical performance have been made [21, 22]. Therefore, coaches tend to overlook late maturing players, having a strong preference for selecting early maturing players as they augment the chances to win matches [23].

Although it has been previously documented that in lower competitive levels (U9-U10), early maturing players revealed greater playing time (PT) than mature late, in higher competitive levels (> U11) this was not clear [24]. Notwithstanding the attempt to reveal the influence of ML on PT, there is still limited evidence regarding these patterns [24]. Also, to the best of our knowledge, there is a lack of evidence supporting the influence of PT and maturational levels on physical and physiological levels between early and normal maturing players.

As football sessions and other training stimuli may influence the concentration levels of GH and IGF1 during adolescence, and knowing that chronic training load exposure may elicit increased GH concentrations [22, 25], it is hypothesized that players with more PT may present greater physical and physiological levels than players with lesser PT. Given the fact that male athletes advanced in maturity levels possess greater strength capabilities, it is expected that they present greater jump performance. While, it is also hypothesized that normal maturing players have the better aerobic capacity, as they seem to perform better in intermittent tasks [7], such as the YYIR1 test.

Considering that in a youth football team there are great development differences between-players in the same team [4], it is expected that this factor is manifested by greater physical qualities differences in the same team. Given that, it is of utmost importance to analyze the within-team maturational status for determining players' physical capacity. Understanding the influence that PT and maturational levels have on the physical and physiological development of early and normal mature athletes may be an optimal strategy to sensibilize coaches to plan training considering these factors. From a practical perspective, this is of paramount importance to make football practice sessions fair and motivating for youth. For these reasons, the purposes of this study were: (i) to describe the fitness status, and hormonal indices according to maturation and competitive level (PT); and (ii) to analyze the comparisons between early and normal mature players (within each competitive level) for the measures of minutes playing, $\mathrm{VO}_{2 \max }$, seven repeated sprint test (7RST), fatigue index, CMJ, GH, and IGF-1 level.

\section{Materials and methods}

\section{Participants}

Twenty-four youth footballers (Mean \pm standard deviation (SD)); chronological age $15.6 \pm 0.2$ years; height $176.4 \pm 5.3 \mathrm{~cm}$; body mass $60.6 \pm 8.3 \mathrm{~kg}$; skeletal age $16.6 \pm 0.8$ years; maturity offset $2.0 \pm 0.4$ years; minutes of playing $1383.4 \pm 260.7 \mathrm{~min}$ ) participated in this study. Players were playing in the Regional Premier League of U16. Positions and number of players in each position were: 2 goalkeepers, 9 defenders, 5 central midfielders, 4 wingers, and 4 attackers. The inclusion criteria were: (1) at 
least playing football for 3 years; (2) they banned for using any kind of food supplement which could affect on growth; and (3) do not participate in any extra training outside this study. The exclusion criteria were: (1) participating in less than $80 \%$ of training and games (formal and no formal); (2) absence in any examination or tests of this study; (3) late maturity status. This study had an Ethics code from the University of Isfahan, also we followed all ethics rights recommendations of the Helsinki Declaration. Before starting the study all players and parents were aware of the benefits and risks of this study and signed a permission letter.

\section{Experimental approach to the problem}

This study was performed as a quasi-experimental and cohort research which was conducted on a cross-sectional basis, the results will be practical. The participants were divided into two groups in terms of PT. The first group played less than $50 \%$ of the season games (PT1), and the second group played more than $50 \%$ of the season games (PT2). Furthermore, other grouping was used based on the maturity level of the players, which were considered as two categories of normal maturity (ML1) and early maturity (ML2) [7]. For grouping players, we used the subtraction of skeletal age and chronological age, if it was more than +1 we called them early mature, and if it was between -1 and +1 we called them on time. All minutes of formal and informal games were included in the variable of minutes playing by the players. At the end of the season after three days' rest the assessment was measured during four days and in the morning, $t$ in the first day, we did blood sampling, X-Ray, and anthropometric measurement for identifying the maturity status of the player. Furthermore, physical fitness tests used in this study were CMJ (second day), the 7RST (third day), and the YYIR1 Test (fourth days). All of these tests were performed in the morning, under the same weather conditions (21-23 ${ }^{\circ} \mathrm{C}$ temperature and 50\% humidity) [26]. Timetable of this study is in Table 1. Participants completed an average of 188-200 training sessions during the season for 8 months, including 43 matches ( 21 official +12 nonofficials) and no long-term injury reports ( 7 players' minor sports injuries) during the course.

\section{Measurements}

The tests in this study were performed in 4 days, at the same temperature ( $21-23{ }^{\circ} \mathrm{C}$ and $50 \%$ humidity) [26], participants stopped physical activity 3 days before the tests.

Blood sampling On the first day, blood samples were taken from the vein of the front arm, after $12 \mathrm{~h}$ of fasting. To minimize measurement error, all blood samples were taken at 8 a.m. by a skilled expert. To measure serum IGF-1and GH levels, the Chemiluminescence method and IMMULITE system were used. Blood samples were centrifuged and kept at $-70^{\circ} \mathrm{C}$ until analyzing.

Skeletal age The EOS Imaging device was used for posterior-anterior imaging of the hand-wrist graph, which is a new device in radiography, where the radiation dose is much lower than previous devices [27-29], and it has also better picture quality [30, 31]. Two-dimensional graph of the EOS Imaging system was used for the determination of skeletal age. This assessment was done by an experienced person, he used the Fels method. The Fels method is one of the three methods for assessing skeletal age from radiography, which is known as the most valid method [10].

Anthropometric measurements Finally, on the first day, anthropometric characteristics were measured [32], the participants stood without shoes, with their shoulders upright and in a normal position, then their standing height was measured. The sitting height was calculated when they sat on a 50-cm bench, with their shoulders and buttocks directly attached to the stadiometer. To measure standing and sitting height, the German SECA device was used with an accuracy of $0.05 \mathrm{~m}$, and to measure the weight of the German SECA 813 device with an accuracy of $0.1 \mathrm{~kg}$. On the other hand, anthropometric measurements were used to maturity offset and age at PHV [11]: Maturity offset $=-9.236+0.0002708$ (leg length $\times$ sitting height $)-0.001663 \quad($ age $\times$ leg length $)+0.007216$ (age $\times$ sitting height $)+0.02292$ (Weight by Height ratio), $R=0.94, R 2=0.891$, and $\mathrm{SEE}=0.592$ ) and for leg length $=$ Standing Height $(\mathrm{cm})-$ Sitting height (cm) [11].

Table 1 Control and assessment period during season

\begin{tabular}{|c|c|c|c|c|c|c|c|c|c|}
\hline \multirow{2}{*}{$\begin{array}{l}\text { Year } \\
\text { Month }\end{array}$} & \multicolumn{4}{|l|}{2018} & \multicolumn{4}{|l|}{2019} & \multirow[t]{3}{*}{ Total } \\
\hline & August September & October & November & December & January & February & March & End of March & \\
\hline Phase & $\begin{array}{l}\text { First preparation } \\
\text { phase ( } 8 \text { week) }\end{array}$ & Regional & games & & $\begin{array}{l}\text { Best of } \\
\text { south } \\
\text { region }\end{array}$ & $\begin{array}{l}\text { Second prepara- } \\
\text { tion phase } \\
(6 \text { week })\end{array}$ & $\begin{array}{l}\text { Best } \\
\text { of Iran } \\
\text { (National) }\end{array}$ & Assessment & \\
\hline Official games no & & 4 & 4 & 4 & 5 & & 4 & 4 days & 21 \\
\hline Non official games no & 4 & & & & & 6 & & & 12 \\
\hline
\end{tabular}


Physiological tests On the second day of the test, after 15 min of a standardized warm-up, which included jogging, stretching, explosive exercises, similar to the test, under the supervision of a conditioning trainer, players performed the CMJ test without swinging the hand, with the hand on the lateral part of the above pelvis for measurements of lower body explosive power [33]. Following the 90 degrees of flexion of the knee, they performed their jump with a maximum power by command of the tester. Two jumps were performed with 5 min' recovery between them, then the best performance was recorded. An electronic pad, the Newest Power Timer company made in Finland was used for testing.

On the third day, the players participated in the 7RST, which included seven $34.5-\mathrm{m}$ sprints with a 25 -s recovery time between them, it assessed anaerobic power [34]. The protocol was started, with a warm-up that included jogging, stretching, and short sprints. After a 15-min warm-up, players were instructed how they should have performed. For identifying the fatigue index we subtracted the worse performance to best performance [35]. To assess the time of each of the players' performances we used Newtest Power Timer device made by Finland company and the $25 \mathrm{~s}$, recovery time was calculated by a stopwatch. The player was notified at the end of each recovery period for the next run, and players were encouraged during the performance.

On the last day of measurement, the aerobic capacity of the players was assessed by the YYIR 1 test. The test started with $40 \mathrm{~m}$ running where players run a 20 -m back and forth, with $10 \mathrm{~m}$ of walking recovery, which is a $5-\mathrm{m}$ back and forth. In this test, with the sound of each beep, the players must be at the exact point of the test, and each level of the test increased, $0.5 \mathrm{~km} / \mathrm{h}$ progressively. Players continued the test until the players could not reach the exact point two times, the last level that the player was able to perform was registered as his record. The following formula was used to determine the $\mathrm{VO}_{2 \max }$. $\mathrm{VO}_{2 \max }\left(\mathrm{ml} \mathrm{kg}^{-1} \mathrm{~min}^{-1}\right)=\mathrm{IR} 1$ distance $(\mathrm{m}) \times 0.0084+36.4[12]$.

Football training In scientific literature, there is a term called the special sport training, and because this research is in the field of football, so we examined the duration of football training, which indicates the time that the player has been engaged in football from the beginning of his life in months. For this purpose, the history of each player was checked from the archive and database of the club, and after that, for more accuracy and precision, the available results were reviewed again with each player and recorded as "Football training" in "months". The average is in Table 1.

Content of each training session The training program for this age group was as follows: 5 training sessions of $90 \mathrm{~min}$ per week, including $10 \mathrm{~min}$ of warm-up, $20 \mathrm{~min}$ of physical training, $10 \mathrm{~min}$ of technical training, $20 \mathrm{~min}$ of tactical training, 25 min of training game (including playing in the small-sided game) and at the end there was a recovery for $5 \mathrm{~min}$. The time of the matches in this age group was $90 \mathrm{~min}$ per game, which was held on the official field according to the rules of the Football Federation of the country for this age group. Training goals in the age group include goals in the small-sided game (development of ball possession, ball transition in speed and rapid organization of zonal defense, retreat and recovery), tactical goals (using defensive and offensive principles quickly), technical goals (focus on passing and controls skills, as well as ball control in small and large spaces) and physical fitness goals (development of aerobic power, linear speed, and explosive power) and were applied in the exercises of each session.

\section{Statistical method}

Mean and SD of variables were used for descriptive statistics. After examining the normality of the test data (Smirnoff Kolmogorov) and homogeneity of variances (Leven test), the Multivariate Analysis of Variance (MANOVA) test, to contrast athletes of early and normal maturity, in both groups of players, PT1 and PT2 were used. Furthermore, Multivariate analyses of covariance (MANCOVA) for controlling contending factor apply two time; first, minutes of playing and second, ML as a covariate, also partial eta-square $\left(\eta p^{2}\right)$ were used to show the magnitude effect size (trivial $<0.2$, small $\geq 0.2$; medium $\geq 0.5$; large $\geq 0.8$ and above) all variables were examined at a significance level of 0.05 using the SPSS 22.

\section{Results}

Table 2 presents the descriptive characteristics of this study sample in Mean \pm SD in both ML and PT groups. We summarized some variables like total season playing time: $1383.4 \pm 260.7 \mathrm{~min}$; height: $176.4 \pm 5.3 \mathrm{~cm} ; \mathrm{VO}_{2 \max }$ : $49.5 \pm 3.5 \mathrm{ml} \cdot \mathrm{kg}^{-1} \cdot \mathrm{min}^{-1}$; IGF-1 level: $392.0 \pm 107.7 \mathrm{ng} / \mathrm{dl}$; skeletal age: $16.6 \pm 0.8$, that was used to divided players in the two groups.

The results of MANOVA are presented in Table 3. Significant differences between PT1 and PT2 were found for minutes of playing $\left(F=45.1, p<0.01, \eta p^{2}=0.7\right)$, age at PHV $\left(F=8.1, p<0.01, \eta p^{2}=0.3\right)$, maturity offset $(F=11.2$, $\left.p<0.01, \eta p^{2}=0.4\right)$, standing height $(F=6.0, p<0.01$, $\left.\eta p^{2}=0.2\right)$, sitting height $\left(F=8.5, p<0.01, \eta p^{2}=0.3\right)$, $\mathrm{VO}_{2 \max }\left(F=9.8, p<0.01, \eta p^{2}=0.3\right), \mathrm{CMJ}(F=9.2, p<0.01$, $\left.\eta p^{2}=0.3\right)$ and IGF-1 level $\left(F=17.0, p<0.01, \eta p^{2}=0.5\right)$. Also, in maturation level groups there were significant differences between early and on time mature players for skeletal age $\left(F=29.0, p<0.01, \eta p^{2}=0.6\right)$, weight $(F=7.5$, $\left.p<0.01, \eta p^{2}=0.3\right), \mathrm{VO}_{2 \max }\left(F=5.2, p<0.05, \eta p^{2}=0.2\right)$, 
Table 2 Descriptive statistics of playing time and maturity groups in inter-variability of U16 youth Football players

\begin{tabular}{|c|c|c|c|c|c|}
\hline \multirow[t]{3}{*}{ Variables } & \multirow{3}{*}{$\begin{array}{l}\text { Total } \\
n=24\end{array}$} & \multicolumn{2}{|c|}{ Playing time groups } & \multicolumn{2}{|c|}{ Maturation level groups } \\
\hline & & PT1 & PT2 & ML1 & ML2 \\
\hline & & $n=12$ & $n=12$ & $n=10$ & $n=14$ \\
\hline Minutes playing (min) & $1383.4 \pm 260.7$ & $1166.8 \pm 170.1$ & $1600 \pm 104.5$ & $1509.0 \pm 256.6$ & $1293.7 \pm 232.2$ \\
\hline Chorological age (year) & $15.6 \pm 0.2$ & $15.66 \pm 0.2$ & $15.57 \pm 0.3$ & $15.6 \pm 0.2$ & $15.6 \pm 0.3$ \\
\hline Skeletal age (year) & $16.6 \pm 0.8$ & $17.0 \pm 0.8$ & $16.3 \pm 0.8$ & $15.9 \pm 0.6$ & $17.2 \pm 0.5$ \\
\hline Football training (month) & $79.9 \pm 19.5$ & $77.8 \pm 19.7$ & $82.0 \pm 19.9$ & $80.3 \pm 15.2$ & $79.6 \pm 22.6$ \\
\hline Age at PHV (year) & $13.6 \pm 0.3$ & $13.4 \pm 0.4$ & $13.8 \pm 0.1$ & $13.7 \pm 0.3$ & $13.6 \pm 0.3$ \\
\hline Maturity offset (year) & $2.0 \pm 0.4$ & $2.2 \pm 0.4$ & $1.7 \pm 0.3$ & $1.9 \pm 0.3$ & $2.0 \pm 0.5$ \\
\hline Standing height $(\mathrm{cm})$ & $176.4 \pm 5.3$ & $179.2 \pm 5.4$ & $173.7 \pm 3.8$ & $175.5 \pm 4.5$ & $177.1 \pm 6.0$ \\
\hline Sitting height $(\mathrm{cm})$ & $93.1 \pm 2.6$ & $94.5 \pm 2.8$ & $91.6 \pm 1.3$ & $92.8 \pm 2.6$ & $93.2 \pm 2.7$ \\
\hline Weight (kg) & $60.6 \pm 8.3$ & $63.6 \pm 10.1$ & $57.7 \pm 4.6$ & $55.7 \pm 5.7$ & $64.2 \pm 8.1$ \\
\hline $\mathrm{VO}_{2 \max }\left(\mathrm{ml} \mathrm{kg}^{-1} \mathrm{~min}^{-1}\right)$ & $49.5 \pm 3.5$ & $47.3 \pm 1.8$ & $51.7 \pm 3.4$ & $51.7 \pm 3.2$ & $47.9 \pm 2.8$ \\
\hline $7 \mathrm{RST}(\mathrm{s})$ & $6.7 \pm 0.2$ & $6.7 \pm 0.3$ & $6.7 \pm 0.2$ & $6.6 \pm 0.2$ & $6.8 \pm 0.3$ \\
\hline Best of 7RST (s) & $6.4 \pm 0.2$ & $6.5 \pm 0.2$ & $6.4 \pm 0.3$ & $6.4 \pm 0.2$ & $6.5 \pm 0.2$ \\
\hline Worse of 7RST (s) & $7.0 \pm 0.3$ & $7.0 \pm 0.4$ & $7.0 \pm 0.3$ & $6.9 \pm 0.3$ & $7.1 \pm 0.3$ \\
\hline Fatigue Index (s) & $0.5 \pm 0.3$ & $0.5 \pm 0.2$ & $0.6 \pm 0.3$ & $0.5 \pm 0.2$ & $0.7 \pm 0.3$ \\
\hline $\mathrm{CMJ}(\mathrm{cm})$ & $40.8 \pm 4.4$ & $38.0 \pm 3.9$ & $43.6 \pm 3.0$ & $43.2 \pm 2.4$ & $39.1 \pm 4.8$ \\
\hline GH level (ng/dl) & $1.6 \pm 1.9$ & $1.1 \pm 1.6$ & $2.3 \pm 2.2$ & $2.1 \pm 1.9$ & $1.3 \pm 1.9$ \\
\hline IGF1 level (ng/dl) & $392.0 \pm 107.7$ & $317.8 \pm 66.4$ & $466.3 \pm 88.5$ & $459.0 \pm 93.3$ & $343.5 \pm 91.8$ \\
\hline
\end{tabular}

$P T 1$ group of player who play less than 50 percent of total season, $P T 2$ group of player who play more than 50 percent of total season, $M L 1$ the group of player who were on normal maturation, $M L 2$ the group of player who were early maturation, $P H V$ Peak Height Velocity, $V O_{2 m a x}$ maximal oxygen uptake, 7RST 7 Repeated Sprint Test, CMJ Countermovement Jump, GH Growth Hormone, IGF1 Insulin-like Growth factor-1

Table 3 Results of MANOVA to test the effect of Playing Time and maturity groups in inter-variability of U16 youth Football players

\begin{tabular}{|c|c|c|c|c|c|c|c|c|c|}
\hline \multirow[t]{2}{*}{ Variables } & \multicolumn{3}{|c|}{ Effects of playing time } & \multicolumn{3}{|c|}{ Effects of maturation level } & \multicolumn{3}{|c|}{ Effects of playing $\times$ maturation } \\
\hline & $F$ & $p$ & $\eta p^{2}$ & $F$ & $p$ & $\eta p^{2}$ & $F$ & $p$ & $\eta p^{2}$ \\
\hline Minutes playing (min) & 45.1 & $<0.001 *$ & 0.7 & 1.2 & 0.283 & 0.1 & 0.7 & 0.413 & $<0.001$ \\
\hline Chorological age (year) & 0.8 & 0.382 & $<0.001$ & 0.1 & 0.330 & 0.1 & 1.8 & 0.199 & 0.1 \\
\hline Skeletal age (year) & 0.5 & 0.493 & $<0.001$ & 29.0 & $<0.001 *$ & 0.6 & 0.5 & 0.476 & $<0.001$ \\
\hline Football training (month) & 0.4 & 0.516 & $<0.001$ & 0.0 & 0.843 & $<0.001$ & 0.7 & 0.430 & $<0.001$ \\
\hline Age at PHV (year) & 8.1 & $0.010^{*}$ & 0.3 & 0.3 & 0.590 & $<0.001$ & 1.5 & 0.242 & 0.1 \\
\hline Maturity offset (year) & 11.2 & $0.003 *$ & 0.4 & $<0.001$ & 0.942 & $<0.001$ & 4.7 & $0.043 *$ & 0.2 \\
\hline Standing height $(\mathrm{cm})$ & 6.0 & $0.024 *$ & 0.2 & $<0.001$ & 0.972 & $<0.001$ & 1.0 & 0.327 & 0.1 \\
\hline Sitting height $(\mathrm{cm})$ & 8.5 & $0.009 *$ & 0.3 & 0.2 & 0.639 & 0.0 & 1.9 & 0.180 & 0.1 \\
\hline Weight (kg) & 0.5 & 0.483 & $<0.001$ & 7.5 & $0.012 *$ & 0.3 & 4.4 & $0.049 *$ & 0.2 \\
\hline $\mathrm{VO}_{2 \max }\left(\mathrm{ml} \mathrm{kg}^{-1} \min ^{-1}\right)$ & 9.8 & $0.005^{*}$ & 0.3 & 5.2 & $0.034 *$ & 0.2 & $<0.001$ & 0.927 & $<0.001$ \\
\hline 7RST (s) & 0.1 & 0.713 & $<0.001$ & 2.5 & 0.129 & 0.1 & 0.8 & 0.370 & $<0.001$ \\
\hline Best of 7RST (s) & 0.1 & 0.779 & $<0.001$ & 1.2 & 0.275 & 0.1 & 0.9 & 0.362 & $<0.001$ \\
\hline Worse of 7RST (s) & 1.7 & 0.206 & 0.1 & 2.1 & 0.100 & 0.1 & 0.9 & 0.355 & $<0.001$ \\
\hline Fatigue Index (s) & 1.6 & 0.220 & 0.1 & 0.6 & 0.454 & $<0.001$ & $<0.001$ & 0.858 & $<0.001$ \\
\hline $\mathrm{CMJ}(\mathrm{cm})$ & 9.2 & $0.007 *$ & 0.3 & 4.4 & $0.048^{*}$ & 0.2 & 4.5 & $0.046^{*}$ & 0.2 \\
\hline GH level (ng/dl) & 1.6 & 0.223 & 0.1 & 0.2 & 0.647 & $<0.001$ & $<0.001$ & 0.845 & $<0.001$ \\
\hline IGF1 level (ng/dl) & 17.0 & $0.001 *$ & 0.5 & 4.9 & $0.039 *$ & 0.2 & 0.1 & 0.333 & 0.5 \\
\hline
\end{tabular}

PHV Peak Height Velocity, $V O_{2 \max }$ maximal oxygen uptake, $7 R S T 7$ Repeated Sprint Test, $C M J$ Counter Movement Jump, GH Growth Hormone, IGF1 Insulin-like Growth Factor-1

*Significant difference at $(p \leq 0.05)$ 
CMJ $\left(F=4.4, p<0.05, \eta p^{2}=0.2\right)$ and IGF-1 level $(F=4.9$, $\left.p<0.05, \eta p^{2}=0.2\right)$. The interaction of groups of PT and their maturation $(\mathrm{PT} \times \mathrm{ML})$ showed that maturity offset $\left(F=4.7, p<0.05, \eta p^{2}=0.2\right)$, weight $(F=4.4, p<0.05$, $\left.\eta p^{2}=0.2\right)$ and CMJ $\left(F=4.5, p<0.05, \eta p^{2}=0.2\right)$ were significantly different.

A MANCOVA was performed to test the effect of PT and ML in inter-variability of U16 youth footballers, and the results are shown in Table 4 . When controlling ML, skeletal age as a covariate, just the parameter of fatigue index $\left(F=47, p<0.05, \eta p^{2}=0.2\right)$ was significant.

\section{Discussion}

The purposes of the present study were to describe the fitness and hormonal status according to ML and PT, and to analyze the differences between ML1 and ML2 within PT1 and PT2 groups, for the measures of aerobic capacity, anaerobic power, power performance, and hormonal concentrations. Results revealed significant differences between both PT and ML groups for maturational status, aerobic capacity, power performance and for IGF1 concentrations. Also, the interaction of PT and ML revealed significant differences for maturity offset and power performance. However, after controlling the ML, using the skeletal age as a covariant, the latter significant differences were reduced only to the fatigue index measure.

The first aim of the present study was to describe the fitness and hormonal levels of both PT1, PT2, ML1, and ML2 groups. Results revealed the greater overall physical performance and greater hormonal mean values for PT2 and ML12? groups, than PT1 and ML21? groups. The characterization of fitness and hormonal measures of young footballers players have previously been made [21, 36, 37]. For instance, in a recent study, it was revealed that footballers had greater changes during 2 seasons in physical and hormonal parameters than non-athletic adolescents from the same chronological age as in the present study [21]. Also, the same study revealed that $\mathrm{GH}$ concentrations of $2.2 \mathrm{ng} /$ dl were similar to our study. In contrast, the IGF1 mean concentration was lower in our study compared to that found in the study of Hammami et al. [21], which showed IGF1 concentrations of $870 \mathrm{ng} / \mathrm{dl}$. In fact, IGF1 values of our sample were similar to the non-athletic population of the above-mentioned study. However, those differences may be due to the divergent methodologies used, as well as the type, duration, and frequency of training that was not controlled in the present study.

Regarding the second aim of the present study, a MANOVA was executed to test the effects of PT and ML on between-group differences of the analyzed response variables. Results revealed that PT influenced the $\mathrm{VO}_{2 \max }$, CMJ, and IGF1 measures, with the PT2 group presenting the greatest values. Other studies revealed that long exposures to football training results in enhanced physical capacities [21, 38]. Indeed, Nobari et al. [16] showed that accumulated training loads influenced the significant differences found at different assessment timings for aerobic capacity, anaerobic power, fatigue index, and maximal speed. Also, despite acute training doses may cause a suppression of IGF1 levels, it seems that chronic exposures to training elicit increases in both GH and IGF1 hormones, that have a great impact on growth and physical performance [22]. For those reasons, it is suggested that players with more PT during a full season show greater physical performance and anabolic hormonal
Table 4 Results of MANCOVA to control playing time and maturation level of U16 youth football players

\begin{tabular}{|c|c|c|c|c|c|c|}
\hline \multirow[t]{2}{*}{ Variables } & \multicolumn{3}{|c|}{ Playing time as covariate } & \multicolumn{3}{|c|}{ Skeletal age as covariate } \\
\hline & $F$ & $p$ & $\eta p^{2}$ & $F$ & $p$ & $\eta p^{2}$ \\
\hline Football training (month) & 0.9 & 0.355 & $<0.001$ & 0.1 & 0.831 & $<0.001$ \\
\hline Standing height $(\mathrm{cm})$ & 0.7 & 0.414 & $<0.001$ & 0.5 & 0.489 & $<0.001$ \\
\hline Sitting height $(\mathrm{cm})$ & 0.4 & 0.551 & $<0.001$ & 0.1 & 0.787 & $<0.001$ \\
\hline Weight $(\mathrm{kg})$ & 4.4 & 0.051 & 0.2 & 0.9 & 0.358 & 0.1 \\
\hline $\mathrm{VO}_{2 \max }\left(\mathrm{ml} \mathrm{kg}^{-1} \min ^{-1}\right)$ & 1.8 & 0.199 & 0.1 & 0.6 & 0.439 & $<0.001$ \\
\hline 7RST (s) & 0.5 & 0.494 & $<0.001$ & 0.2 & 0.659 & $<0.001$ \\
\hline Best of 7RST (s) & 1.1 & 0.315 & 0.1 & 1.8 & 0.200 & 0.1 \\
\hline Worse of 7RST (s) & 0.4 & 0.548 & $<0.001$ & 0.7 & 0.425 & $<0.001$ \\
\hline Fatigue Index (s) & $<0.001$ & 0.987 & $<0.001$ & 4.7 & $0.043^{*}$ & 0.2 \\
\hline $\mathrm{CMJ}(\mathrm{cm})$ & $<0.001$ & 0.855 & $<0.001$ & 0.9 & 0.356 & 0.1 \\
\hline GH level (ng/dl) & 1.3 & 0.263 & 0.1 & $<0.001$ & 0.962 & $<0.001$ \\
\hline IGF1 level (ng/dl) & 1.5 & 0.232 & 0.1 & 1.1 & 0.317 & 0.1 \\
\hline
\end{tabular}

PHV Peak Height Velocity, $V O_{2 \max }$ maximal oxygen uptake, $7 R S T 7$ Repeated Sprint Test, $C M J$ Counter Movement Jump, GH Growth Hormone, IGF1 Insulin-like Growth Factor-1

*Significant difference at $(p \leq 0.05)$ 
concentrations. Given that, special attention should be given to players with less PT, thus, it would be of paramount importance for coaches to promote a between-player balanced training stimulus.

Although there is a lack of studies investigating the effects of PT on the analyzed dependent variables in the present study, other studies attempted to test the influence of physical and hormonal measures, and maturational status on PT [24, 39]. In fact, Deprez et al. [39] showed that jump performance influenced approximately $17 \%$ of the variance in future PT. Also, Goto et al. [24] revealed that early mature players had more PT than their late-maturing counterparts in lower age categories. This was not observed in the present study, as the ML2 group, early maturing players showed slightly lesser minutes of play, but not representing significant differences. However, our sample is from higher age categories (U-16), and the above-mentioned study [24], highlighted that at higher age categories the differences between early and late maturing players were not clear. Given that, more studies should be conducted for ensuring greater information about these types of influences of PT on physical, physiological, and hormonal measures, and vice-versa.

Furthermore, ML showed effects on player's weight, $\mathrm{VO}_{2 \max }, \mathrm{CMJ}$, and IGF1 measures, being the ML1 group the lighter, and with the greatest values of $\mathrm{VO}_{2 \max }, \mathrm{CMJ}$, and IGF1. Thus, after removing the influence of skeletal age, used as a covariate, from the analysis, the significant differences were reduced to fatigue index only. Indeed it has been documented that the ML of footballers are strongly associated with greater physical performance [7, 40]. Greater development of aerobic capacity is shown at the PHV, and greater maximal aerobic power, strength, and power development might occur slightly later [38, 41, 42]. Also, as children advance in maturity status during puberty, it is expected a greater activity of operating hormones such as the GH and IGF1 concentrations, which facilitates human growth [22, 37]. Thus, when footballers are at critical stages of puberty, it is expected that they become more heavy and taller, confirming our results considering the effects of ML1 on player's weight. Coaches should be aware of these effects as they may assume a preponderant role when considering the selection and PT.

The present study has some limitations. One of the main limitations is the small size of the sample, which include only one U16 football team. However, this is a common limitation considering elite sports contexts. Another limitation is related to the fact that we did not consider any external load and heart rate-based measures [43-45]. It would be of interest that future studies focused on the influence that PT and ML might have on different heart rate and external variables. Despite those limitations, the present study showed that both PT and maturation status may influence the level of anthropometric measures, aerobic capacity, power performance, and IGF1 concentrations. Given that, coaches must provide balanced PT opportunities within a youth team, and individualize training to promote greater physical, physiological and hormonal adaptations to the whole team players.

\section{Conclusions}

In short, significant differences between both PT and ML groups for maturational status, aerobic capacity, power performance, and for IGF1 concentrations were found. PT and ML together revealed significant differences for maturity offset and power performance. Considering the present study findings, it is suggested that the above-mentioned measures seem to be influenced by PT and ML, as after controlling the skeletal age as a covariant, the latter significant differences were reduced only to the fatigue index measure.

Funding Open Access funding provided thanks to the CRUE-CSIC agreement with Springer Nature.

\section{Declarations}

Conflict of interest The authors declare that they have no conflict of interest.

Ethics approval This study had an Ethics code from the University of Isfahan, also we followed all ethics rights recommendations of the Helsinki Declaration. Before starting the study all players and parents were aware of the benefits and risks ofthis study and signed a permission letter (ie., consent form).

Informed consent Informed consent was obtained from all subject and their parents.

Open Access This article is licensed under a Creative Commons Attribution 4.0 International License, which permits use, sharing, adaptation, distribution and reproduction in any medium or format, as long as you give appropriate credit to the original author(s) and the source, provide a link to the Creative Commons licence, and indicate if changes were made. The images or other third party material in this article are included in the article's Creative Commons licence, unless indicated otherwise in a credit line to the material. If material is not included in the article's Creative Commons licence and your intended use is not permitted by statutory regulation or exceeds the permitted use, you will need to obtain permission directly from the copyright holder. To view a copy of this licence, visit http://creativecommons.org/licenses/by/4.0/.

\section{References}

1. Sarmento H, Marcelino R, Anguera MT, Campaniço J, Matos N, Leitão JC (2014) Match analysis in football: a systematic review. J Sports Sci 32(20):1831-43. http://www.ncbi.nlm.nih.gov/pub$\operatorname{med} / 24787442$ 
2. Dellal A, Lago-Peñas C, Rey E, Chamari K, Orhant E (2015) The effects of a congested fixture period on physical performance, technical activity and injury rate during matches in a professional soccer team. Br J Sports Med 49(6):390-394. https://doi.org/10. 1136/bjsports-2012-091290

3. Barnes C, Archer D, Hogg B, Bush M, Bradley P (2014) The evolution of physical and technical performance parameters in the English premier league. Int J Sports Med 35(13):1095-1100. https://doi.org/10.1055/s-0034-1375695

4. Sarmento H, Anguera MT, Pereira A, Araújo D (2018) Talent identification and development in male football: a systematic review. Sport Med 48(4):907-931. https://doi.org/10.1007/ s40279-017-0851-7

5. Till K, Cobley S, O’Hara J, Chapman C, Cooke C (2013) A longitudinal evaluation of anthropometric and fitness characteristics in junior rugby league players considering playing position and selection level. J Sci Med Sport 16(5):438-443

6. Nobari H, Aquino R, Clemente FM, Khalafi M, Adsuar JC, PérezGómez J (2020) Description of acute and chronic load, training monotony and strain over a season and its relationships with wellbeing status: a study in elite under-16 soccer players. Physiol Behav 225:113117. https://linkinghub.elsevier.com/retrieve/pii/ S0031938420304315

7. Malina RM, Rogol AD, Cumming SP, Coelho-E-Silva MJ, Figueiredo AJ (2015) Biological maturation of youth athletes: assessment and implications. Br J Sports Med 49(13):852-859

8. Figueiredo AJ, Coelho-E-Silva MJ, Sarmento H, Moya J, Malina RM (2020) Adolescent characteristics of youth soccer players: do they vary with playing status in young adulthood? Res Sport Med 28(1):72-83. https://doi.org/10.1080/15438627.2019.1586704

9. Vaeyens R, Lenoir M, Williams AM, Philippaerts RM (2008) Talent identification and development programmes in sport: current models and future directions. Sport Med 38(9):703-714

10. Roche AF, Thissen D, Chumlea W (1988) Assessing the skeletal maturity of the hand-wrist: fels method. Thomas

11. Mirwald RL, Baxter-Jones ADG, Bailey DA, Beunen GP (2002) An assessment of maturity from anthropometric measurements. Med Sci Sport Exerc 34(4):689-694

12. Bangsbo J, Iaia FM, Krustrup P (2008) The Yo-Yo intermittent recovery test. Sport Med 38(1):37-51. https://doi.org/10.2165/ 00007256-200838010-00004

13. Tønnessen E, Hem E, Leirstein S, Haugen T, Seiler S (2013) Maximal aerobic power characteristics of male professional soccer players, 1989-2012. Int J Sports Physiol Perform 8(3):323-329

14. Schmitz B, Pfeifer C, Kreitz K, Borowski M, Faldum A, Brand SM (2018) The Yo-Yo intermittent tests: a systematic review and structured compendium of test results. Front Physiol 9(JUL):1-16

15. Slimani M, Nikolaidis PT (2018) Anthropometric and physiological characteristics of male soccer players according to their competitive level, playing position and age group: a systematic review. J Sports Med Phys Fitness 2019:141-163

16. Nobari H, Silva AF, Clemente FM, Siahkouhian M (2020) Analysis of fitness status variations of under-16 soccer players over a season and their relationships with maturational status and training load. Front Physiol. https://doi.org/10.3389/fphys.2020. 597697

17. Nobari H, Tubagi Polito LF, Clemente FM, Pérez-Gómez J, Ahmadi M, Garcia-Gordillo MÁ, et al (2020) Relationships between training workload parameters with variations in anaerobic power and change of direction status in elite youth soccer players. Int J Environ Res Public Health 17(21):7934. https://www. mdpi.com/1660-4601/17/21/7934

18. Nikolaïdis P (2011) Anaerobic Power across Adolescence in Soccer Players. Hum Mov 12(4):342-347. http://www.degruyter.com/ view/j/humo.2011.12.issue-4/v10038-011-0039-1/v10038-0110039-1.xml

19. Buchheit M, Mendez-Villanueva A, Simpson BM, Bourdon PC (2010) Repeated-sprint sequences during youth soccer matches. Int J Sports Med 31(10):709-716. https://doi.org/10.1055/s-00301261897

20. Duarte JP, Coelho-E-Silva MJ, Costa D, Martinho D, Luz LGO, Rebelo-Gonçalves R et al (2019) Repeated sprint ability in youth soccer players: independent and combined effects of relative age and biological maturity. J Hum Kinet 67(1):209-221

21. Hammami MA, Ben Abderrahman A, Rhibi F, Nebigh A, Coppalle S, Ravé G et al (2018) Somatotype hormone levels and physical fitness in elite young soccer players over a two-year monitoring period. J Sport Sci Med 17(3):455-464

22. Eliakim A, Nemet D (2010) Exercise training, physical fitness and the growth hormone-insulin-like growth factor-1 axis and cytokine balance. Med Sport Sci 55:128-140

23. Henriksen K, Stambulova N, Roessler KK (2010) Holistic approach to athletic talent development environments: a successful sailing milieu. Psychol Sport Exerc 11(3):212-222

24. Goto H, Morris JG, Nevill ME (2019) Influence of biological maturity on the match performance of 8- to 16-year-old, elite, male, youth soccer players. J Strength Cond Res 33(11):3078-84. http://journals.lww.com/00124278-201911000-00023

25. Hadzović A, Nakas-Ićindić E, Kucukalić-Selimović E, Avdagić N, Zaciragić A (2004) The level of physical activity and the growth hormone $(\mathrm{GH})$ response to acute physical exercise. Bosn J Basic Med Sci 4(3):47-49

26. Lippincott W, Wilkins (2013) ACSM's guidelines for exercise testing and prescription. Medicine ACoS

27. Ilharreborde B, Ferrero E, Alison M, Mazda K (2016) EOS microdose protocol for the radiological follow-up of adolescent idiopathic scoliosis. Eur Spine J 25(2):526-531

28. Luo TD, Stans AA, Schueler BA, Larson AN (2015) Cumulative radiation exposure with EOS imaging compared with standard spine radiographs. Spine Deform 3(2):144-150

29. Hui SCN, Pialasse JP, Wong JYH, Lam T, Ng BKW, Cheng JCY et al (2016) Radiation dose of digital radiography (DR) versus micro-dose $\mathrm{x}$-ray (EOS) on patients with adolescent idiopathic scoliosis: 2016 SOSORT- IRSSD “John Sevastic Award” Winner in Imaging Research. Scoliosis Spinal Disord 11(1):1-8

30. Wade R, Yang H, McKenna C, Faria R, Gummerson N, Woolacott N (2013) A systematic review of the clinical effectiveness of EOS 2D/3D X-ray imaging system. Eur Spine J 22(2):296-304

31. Deschenes S, Charron G, Beaudoin G, Labelle H, Miron M, Parent S (2016) Diagnostic imaging of spinal deformities: reducing patients' radiation dose. Spine (Phila Pa 1976) 35(9):989-994

32. Arazi H, Mirzaei B, Nobari H (2015) Anthropometric profile, body composition and somatotyping of national Iranian crosscountry runners. Turkish J Sport Exerc 17(2):35

33. Bangsbo J, Mohr M (2012) Fitness testing in football. Bangsbosport

34. Haugen TA, Tønnessen E, Seiler S (2012) Speed and countermovement-jump characteristics of elite female soccer players, 1995-2010. Int J Sports Physiol Perform 7(4):340-349. http:// www.ncbi.nlm.nih.gov/pubmed/22645175

35. Kaplan T (2010) Examination of repeated sprinting ability and fatigue index of soccer players according to their positions. $J$ Strength Cond Res Natl Strength Cond Assoc 24(6):1495-1501

36. Perroni F, Fittipaldi S, Falcioni L, Ghizzoni L, Borrione P, Vetrano M et al (2019) Effect of pre-season training phase on anthropometric, hormonal and fitness parameters in young soccer players. Boullosa D, editor. PLoS ONE 14(11):e0225471. https:// doi.org/10.1371/journal.pone.0225471

37. Nobari H, Kargarfard M, Minasian V, Cholewa JM, Pérez-Gómez J (2021) The effects of 14-week betaine supplementation on 
endocrine markers, body composition and anthropometrics in professional youth soccer players: a double blind, randomized, placebo-controlled trial. J Int Soc Sports Nutr 18(1):20. https:// doi.org/10.1186/s12970-021-00417-5

38. Vänttinen T, Blomqvist M, Nyman K, Häkkinen K (2011) Changes in body composition, hormonal status, and physical fitness in 11-, 13-, and 15-year-old finnish regional youth soccer players during a two-year follow-up. J Strength Cond Res 25(12):3342-3351. http://journals.lww.com/00124278-20111 2000-00016

39. Deprez DN, Fransen J, Lenoir M, Philippaerts RM, Vaeyens R (2015) A retrospective study on anthropometrical, physical fitness, and motor coordination characteristics that influence dropout, contract status, and first-team playing time in high-level soccer players aged eight to eighteen years. J Strength Cond Res 29(6):16921704. http://journals.lww.com/00124278-201506000-00031

40. Lovell R, Fransen J, Ryan R, Massard T, Cross R, Eggers T, et al (2019) Biological maturation and match running performance: a national football (soccer) federation perspective. J Sci Med Sport 22(10):1139-1145. https://linkinghub.elsevier.com/retrieve/pii/ S1440244018309848

41. Philippaerts RM, Vaeyens R, Janssens M, Van Renterghem B, Matthys D, Craen R et al (2006) The relationship between peak height velocity and physical performance in youth soccer players. J Sports Sci 24(3):221-230. https://doi.org/10.1080/0264041050 0189371

42. Nobari H, Alves AR, Clemente FM, Pérez-Gómez J, Clark CCT, Granacher U et al (2021) Associations between variations in accumulated workload and physiological variables in young male soccer players over the course of a season. Front Physiol 12:233. https://doi.org/10.3389/fphys.2021.638180/full

43. Clemente FM, Silva R, Chen Y-S, Aquino R, Praça GM, Castellano J, et al (2021) Accelerometry-workload indices concerning different levels of participation during congested fixture periods in professional soccer: a pilot study conducted over a full season. Int J Environ Res Public Health 18(3):1137. https://www.mdpi. com/1660-4601/18/3/1137

44. Nobari H, Praça GM, Clemente FM, Pérez-Gómez J, Carlos Vivas J, Ahmadi M (2020) Comparisons of new body load and metabolic power average workload indices between starters and nonstarters: a full-season study in professional soccer players. Proc Inst Mech Eng Part P J Sport Eng Technol. https://doi.org/10. $1177 / 1754337120974873$

45. Nobari H, Oliveira R, Brito JP, Pérez-Gómez J, Clemente FM, Ardigò LP (2021) Comparison of running distance variables and body load in competitions based on their results: a full-season study of professional soccer players. Int J Environ Res Public Health 18(4):2077. https://www.mdpi.com/1660-4601/18/4/2077

Publisher's Note Springer Nature remains neutral with regard to jurisdictional claims in published maps and institutional affiliations. 